\title{
Arabidopsis SPCH is a MAPK specificity factor that controls entry into the stomatal lineage
}

\author{
Gregory R. Lampard \\ Department of Biology; Stanford University; Stanford, CA USA
}

Key words: MAPK, stomata, development, SPCH, bHLH

Mitogen activated protein kinase (MAPK) signaling modules that incorporate AtMPK3 and AtMPK6 control critical aspects of Arabidopsis biology including stress responses, development, cell division and cell death. Arabidopsis stomatal development is negatively regulated by the YDA-MKK4/5-MPK3/6 MAPK module and follows a three step pathway of asymmetric and symmetric divisions followed by terminal differentiation. We have identified the bHLH transcription factor SPCH, which controls entry into the stomatal lineage as a substrate of AtMPK3 and AtMPK6. These findings suggest that SPCH activity may be directly affected by environmental conditions to enable the plant to modify stomatal development in response to suboptimal climates.

\section{MAPK Signaling in Arabidopsis}

Mitogen activated protein kinase (MAPK) signaling networks are ubiquitous amongst eukaryotes where they control numerous aspects of biology including developmental processes and stress responses. ${ }^{1,2}$ In plants, MAPKs regulate a range of processes including responses to abiotic and biotic stresses, initiation of hypersensitive-response-like cell death and control of cell division and development. ${ }^{3-5}$ Canonical MAPK signaling modules are comprised of a three kinase-hierarchical cascade that involves the serial phosphorylation and activation of a MAPK kinase kinase (MAPKKK), a MAPK kinase (MAPKK) and finally a MAPK. ${ }^{2}$ Upon activation, plant MAPKs phosphorylate several classes of downstream effector proteins, including cytoskeletal elements, biosynthetic enzymes, protein kinases and transcription factors to evoke specific cellular responses. ${ }^{6}$ Sequencing of the Arabidopsis and other plant genomes, including rice and poplar, revealed

Correspondence to: Gregory R. Lampard; Department of Biology; Stanford University; Stanford, CA USA; Email: glampard@stanford.edu

Submitted: 02/23/09; Accepted: 02/26/09

Previously published online as a Plant Signaling \& Behavior E-publication: http://www.landesbioscience.com/journals/psb/article/8296

Addendum to: Lampard GR, Macalister CA, Bergmann DC. Arabidopsis stomatal initiation is controlled by MAPK-mediated regulation of the $\mathrm{bHLH}$ SPEECHLESS. Science 2008; 322:1113-6; PMID: 19008449; DOI: 10.1126/ science. 1162263. extended gene families of each class of kinase relative to other eukaryotes, with approximately $60 \mathrm{MAPKKKs,} 10 \mathrm{MAPKKs}$ and 20 MAPKs being encoded by the Arabidopsis genome. ${ }^{7,8}$ Being sessile organisms, plants must be able to respond and adapt to a plethora of environmental stimuli. Therefore, it may not be surprising to see these extended gene families in plants; one could speculate that specific combinations of MAPKKKs, MAPKKs and MAPKs would be used to translate the multitude of upstream activating stimuli into discrete cellular responses.

While recent high-throughput studies aimed at identifying the targets of all Arabidopsis MAPKKs and MAPKs have expanded our knowledge of MAPK signaling networks, it is clear that many upstream stimuli converge upon a single MAPK module consisting of the MAPKKs MKK4 and MKK5 and the MAPKs MPK3 and MPK6 (reviewed in ref. 9). These kinases were initially characterized as playing central roles in responses to environmental and mechanical stresses and have since been reported to also be centrally involved in hormone response networks and developmental processes. This raises this question: How can a single MAPK module specifically control this wide array of downstream processes? Methods of controlling MAPK signaling specificity in other eukaryotes include the use of specific scaffold proteins that allow only certain kinases and substrates to come together physically and the discrete spatio-temporal expression of kinases and substrates. 5,10 To date, no MAPK scaffold proteins have been reported in plants and the MKK4/5-MPK3/6 proteins are broadly expressed, suggesting that specificity may largely be controlled through regulation of substrate expression.

\section{MAPK Control of Arabidopsis Stomatal Development}

Arabidopsis stomatal development follows a series of asymmetric and symmetric cell division and differentiation steps that ultimately lead to the formation of a mature stomate (Fig. 1A). The MAPK signaling module consisting of YDA-MKK4/5-MPK3/6 is a general inhibitor of stomatal development, ${ }^{11,12}$ whereas positive regulators include the related bHLH transcription factors SPCH, MUTE and FAMA, which control the entry, progression and terminal differentiation stages of guard cell development, respectively. ${ }^{13-15}$ Genetic evidence placed this MAPK signaling module upstream of entry into the stomatal lineage and the 
Figure 1. (A) MAPK control of stomatal development. Guard cells are formed by a three step process involving asymmetric and symmetric cell divisions (1-3). Progression to each subsequent stage is promoted by SPCH (1; creation of meristemoid (M)), MUTE $(2 ;(M)$ to guard mother cell $(G M C))$ and FAMA (3; GMC to guard cell). Entry into the stomatal lineage is controlled by interaction of unidentified ligands with multiple receptors (ER, ERL I, ERL2 and TMM). This activates the YDA-MKK4/5-MPK3/6 MAPK module, which regulates entry into the stomatal lineage by directly phosphorylating SPCH. Alternatively, abiotic and/or biotic stresses trigger activation of yet to be identified MAPKKKs resulting in activation of MKK4/5 and MPK3/6 which may influence stomatal development patterns. (B) Protein architecture of the bHLH SPCH, which, in addition to the bHLH domain, contains a conserved C-terminus (SFM) and the unique MAPK target domain (MAPKTD). The amino acid sequence of the MAPKTD is shown with MAPK high stringency phosphorylation sites 1-5 depicted in italics. Phosphorylation sites confirmed by MS/MS analysis are depicted in bold.

phenotypes of plants lacking SPCH are the same as plants in which the YDA-MKK4/5-MPK3/6 module is constitutively active-an epidermis completely devoid of guard cells. ${ }^{12,15}$ These data, coupled with the tight regulation of $\mathrm{SPCH}$ expression $(\mathrm{SPCH}$ is only expressed during the MMC to M transition; Fig. 1A), made it a prime candidate to be a specificity factor allowing the MAPK module to control entry into the stomatal lineage. Consistent with this, we identified SPCH as an in vitro substrate of MPK3 and MPK6, making it the first specificity conferring developmental substrate of MPK3 and MPK6. ${ }^{16}$ Specifically, SPCH is capable of being phosphorylated on at least five serine or threonine residues contained within a unique domain referred to as the MAPK target domain (MAPKTD; Fig. 1B). Furthermore, because elimination of the ability of SPCH to be phosphorylated resulted in dramatic stomatal over-proliferation, it is clear that phosphorylation of $\mathrm{SPCH}$ is required to prevent entry into the stomatal lineage.

Intriguingly, an apparent dual role of phosphorylation of $\mathrm{SPCH}$ by MPK3 and MPK6 was identified. It appears that phosphorylation is required both for $\mathrm{SPCH}$ activity (i.e., promoting entry into the stomatal lineage) and inhibition of subsequent SPCH activity (i.e., ensuring correct stomatal patterning). SPCH phosphorylation generally correlates with inhibition of stomatal development yet phosphorylation on S193 is critical for SPCH function. This is reflected by the inability of $\mathrm{SPCH}$ variant proteins containing a non-phosphorylatable alanine residue at position 193 to rescue spch-null plants. A common result of phosphorylation is a change in protein stability. In the case of bHLH transcription factors PIF1, PIF3 and PIF5, phosphorylation immediately precedes protein degradation by the proteosome. ${ }^{17-19}$ Given the stomatal over-proliferation phenotypes observed in non-phosphorylatable $\mathrm{SPCH}$ variants such as the SPCH $\triangle \mathrm{MAPKTD}$ variant, it is tempting to speculate that a predominant role of phosphorylation is the inhibition of $\mathrm{SPCH}$ activity by triggering protein degradation. However, this does not explain the absolute necessity for a SPCH variant containing a phosphorylatable S193 residue for the formation of mature guard cell complexes. The most plausible explanation for this phenomenon is that initial phosphorylation of SPCH on S193 yields an active protein, either by altering the binding affinity of SPCH for target DNA sites or by changing the ability of SPCH to interact with additional bHLH proteins, such as the recently reported SCRM1/2 bHLH proteins. ${ }^{20}$ This

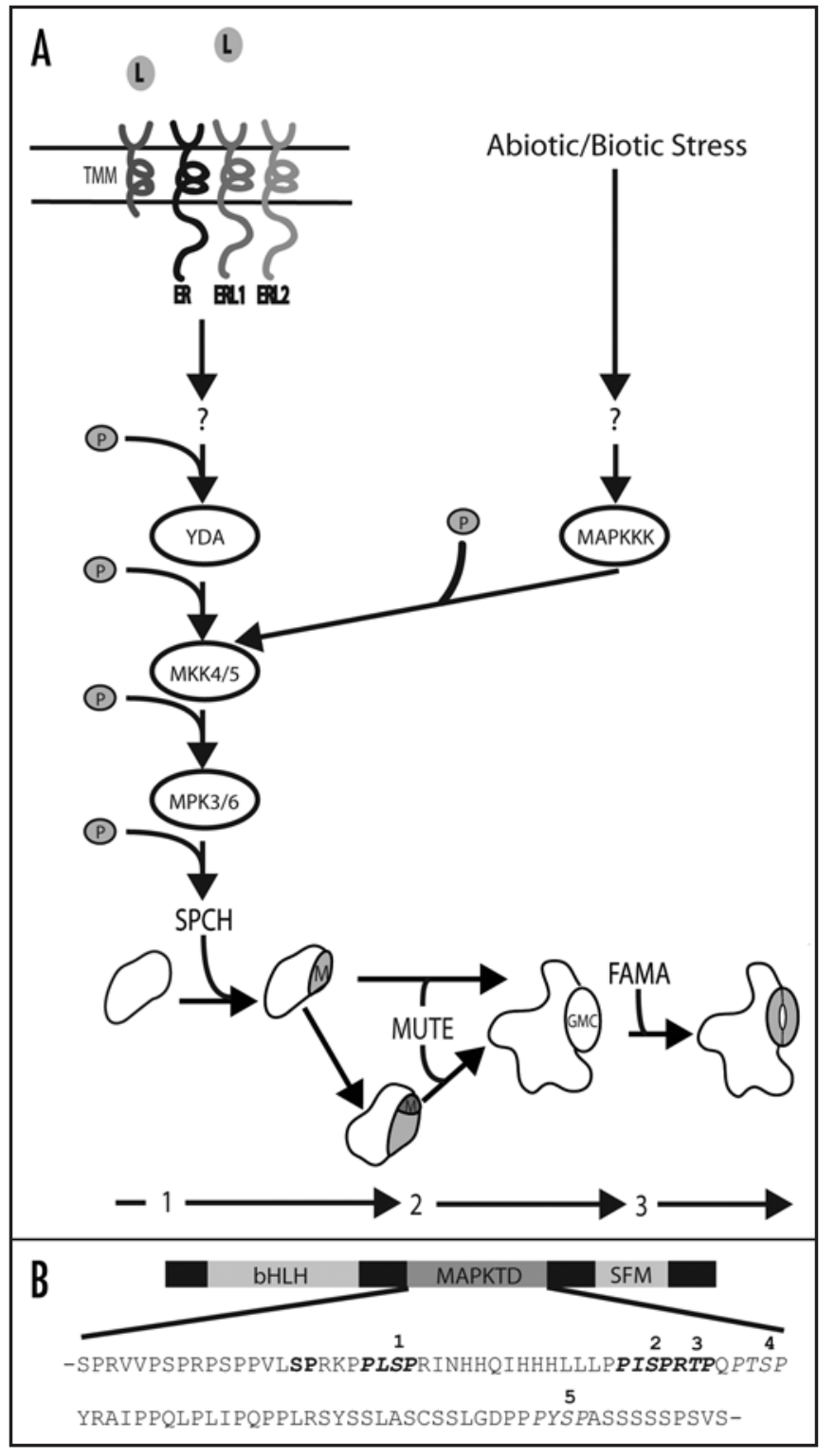

would be somewhat similar to the case of the cyclin kinase inhibitor $\mathrm{p} 27^{\mathrm{kip}}$, where phosphorylation affects protein stability in opposite ways depending upon which residue is phosphorylated. Phosphorylation of T198 enhances protein stability, but phosphorylation of T187 triggers rapid degradation of p27kip1. The coordination of these events with specific stages of the cell cycle allows proper cell cycle progression. ${ }^{21}$

\section{Future Directions}

Clearly, deciphering the properties and kinetics of $\mathrm{SPCH}$ phosphorylation in vivo will yield answers to many outstanding questions. For example, (1) is SPCH initially phosphorylated on S193 and does this enable SPCH activity? (2) Does the rate at which additional serine/threonine residues are phosphorylated correlate with the rate of $\mathrm{SPCH}$ degradation or the severity of inhibition of SPCH activity? (3) Are there differences in the ability 


\section{SPCH is a MPK3/6 specificity factor}

of MPK3 and MPK6 to phosphorylate specific and/or overlapping residues in vivo?

Answering these questions will no doubt shed light onto precisely how phosphorylation of SPCH by MPK3 and MPK6 controls entry into the stomatal lineage, but it may also explain the links between stress and direct control of stomatal development. Datasets from the plethora of publicly available microarrays reveal a trend towards a rapid downregulation of metabolic and developmental genes in response to abiotic and biotic challenges, suggesting that plants can delay developmental processes while responding to particular stresses. Consistent with this hypothesis, environmental challenges such as increased carbon dioxide levels, increased temperatures and changes in light quality all have significant impacts on stomatal densities in Arabidopsis, ${ }^{22,23}$ yet the precise mechanisms controlling these changes have not been reported. Linking the control of stomatal development to a stress-activated MAPK module provides a testable model for how these changes in development might be carried out (Fig. 1A). Experiments could include an examination of stress induced changes in stomatal densities in WT, mpk3 and $m p k 6$ null plants; using mutants lacking one of MPK3 or MPK6 may create hyper- or hyposensitive phenotypes. If future data supports this model, experiments testing how widespread stress-induced control of stomatal development is should be conducted: Stresses such as cold, drought and pathogen attack also lead to rapid activation of MPK3/6 coupled with decreased expression of developmental and metabolic genes.' Again, experiments aimed at answering these questions will clarify these speculations.

From an evolutionary context, linking stomatal development to stress-associated signaling networks is not surprising. Dramatic lifestyle changes are inherent in coping with the transition from an aquatic environment to land. Therefore, yielding control over gas exchange via both structural innovations (stomata) and stressresponsive regulatory modules is logical and efficient. Beyond environmental stresses, MAPKs play significant roles in responding to pathogen attack. Stomata are a major access point for bacterial pathogens such as Pseudomonas syringae and Xanthomonas campestris. ${ }^{24,25}$ Infection by either of these pathogens triggers rapid activation of MPK3/6, which could be coupled with an inhibition of subsequent stomatal development. Each of the above examples suggests that stresses may have the ability to finely control stomatal development through altering MAPK signaling, an area that remains largely unexplored. Clearly we will benefit from the thorough characterization of which and how stresses, both abiotic and biotic, control stomatal development (Fig. 1A).

\section{Conclusions}

Identification of SPCH as a substrate of the stress-activated MAPKs, MPK3 and MPK6 gives further evidence that MAPK signaling may be directly involved in modulating stomatal development patterns in response to fluctuating environmental conditions. Characterization of the consequences of and phosphorylation kinetics of SPCH in vivo will provide details surrounding the mechanisms by which plants are able to respond and adapt to abiotic and biotic challenges which could ultimately lead to the development of more stress tolerant plants.

\section{References}

1. Nakagami H, Pitzschke A, Hirt H. Emerging MAP kinase pathways in plant stress signalling. Trends Plant Sci 2005; 10:339-46.

2. Widmann C, Gibson S, Jarpe MB, Johnson GL. Mitogen-activated protein kinase: Conservation of a three-kinase module from yeast to human. Physiol Rev 1999; 79:143-80.

3. Ichimura K, Shinozaki K, Tena G, Sheen J, Henry Y, Champion A, et al. Mitogenactivated protein kinase cascades in plants: A new nomenclature. Trends Plant Sci 2002; 7:301-8.

4. Pedley KF, Martin GB. Role of mitogen-activated protein kinases in plant immunity. Curr Opin Plant Biol 2005; 8:541-7.

5. Tanoue T, Nishida E. Molecular recognitions in the MAP kinase cascades. Cell Signal 2003; 15:455-62.

6. Popescu SC, Popescu GV, Bachan S, Zhang Z, Gerstein M, Snyder M, Dinesh-Kumar SP. MAPK target networks in Arabidopsis thaliana revealed using functional protein microarrays. Genes Dev 2009; 23:80-92.

7. Hamel L, Nicole M, Sritubtim S, Morency M, Ellis M, Ehlting J, et al. Ancient signals: Comparative genomics of plant MAPK and MAPKK gene families. Trends Plant Sci 2006; 11:192-8.

8. Ichimura K, Shinozaki K, Tena G, Sheen J, Henry Y, Champion A, et al. Mitogenactivated protein kinase cascades in plants: A new nomenclature. Trends Plant Sci 2002; 7:301-8.

9. Colcombet J, Hirt H. Arabidopsis MAPKs: A complex signalling network involved in multiple biological processes. Biochem J 2008; 413:217-26.

10. Whitmarsh AJ, Davis RJ. Structural organization of MAP-kinase signaling modules by scaffold proteins in yeast and mammals. Trends Biochem Sci 1998; 23:481-5.

11. Bergmann DC, Lukowitz W, Somerville CR. Stomatal development and pattern controlled by a MAPKK kinase. Science 2004; 304:1494-7.

12. Wang H, Ngwenyama N, Liu Y, Walker JC, Zhang S. Stomatal development and patterning are regulated by environmentally responsive mitogen-activated protein kinases in Arabidopsis. Plant Cell 2007; 19:63-73.

13. Ohashi-Ito K, Bergmann DC. Arabidopsis FAMA controls the final Proliferation/ Differentiation switch during stomatal development. Plant Cell 2006; 18:2493-505.

14. Pillitteri LJ, Sloan DB, Bogenschutz NL, Torii KU. Termination of asymmetric cell division and differentiation of stomata. Nature 2007; 445:501-5.

15. MacAlister CA, Ohashi-Ito K, Bergmann DC. Transcription factor control of asymmetric cell divisions that establish the stomatal lineage. Nature 2007; 445:537-40.

16. Lampard GR, MacAlister CA, Bergmann DC. Arabidopsis stomatal initiation is controlled by MAPK-mediated regulation of the bHLH SPEECHLESS. Science 2008; 322:1113-6

17. Al-Sady B, Ni W, Kircher S, Schäfer E, Quail PH. Photoactivated phytochrome induces rapid PIF3 phosphorylation prior to proteasome-mediated degradation. Mol Cell 2006; 23:439-46.

18. Shen H, Zhu L, Castillon A, Majee M, Downie B, Huq E. Light-induced phosphorylation and degradation of the negative regulator PHYTOCHROME-INTERACTING FACTOR1 from arabidopsis depend upon its direct physical interactions with photoactivated phytochromes. Plant Cell 2008; 20:1586-602.

19. Shen Y, Khanna R, Carle CM, Quail PH. Phytochrome induces rapid PIF5 phosphorylation and degradation in response to red-light activation. Plant Physiol 2007; 145:1043-51.

20. Kanaoka MM, Pillitteri LJ, Fujii H, Yoshida Y, Bogenschutz NL, Takabayashi J, et al. SCREAM/ICE1 and SCREAM2 specify three cell-state transitional steps leading to Arabidopsis stomatal differentiation. Plant Cell 2008; 20:1775-85.

21. Kossatz U, Vervoorts J, Nickeleit I, Sundberg H, Arthur J, Manns M, Malek N. C-terminal phosphorylation controls the stability and function of $\mathrm{p} 27^{\mathrm{kip} 1}$. EMBO J 2006; 25:5159-70.

22. Casson S, Gray J. Influence of environmental factors on stomatal development. New Phytol 2008; 178:9-23.

23. Casson SA, Franklin KA, Gray JE, Grierson CS, Whitelam GC, Hetherington AM. Phytochrome B and PIF4 regulate stomatal development in response to light quantity. Curr Biol 2009; 19:229-34.

24. Gudesblat GE, Torres PS, Vojnov AA. Xanthomonas campestris overcomes Arabidopsis stomatal innate immunity through a DSF cell-to-cell signal-regulated virulence factor. Plant Physiol 2009; 149:1017-27.

25. Melotto M, Underwood W, He SY. Role of stomata in plant innate immunity and foliar bacterial diseases. Annu Rev Phytopathol 2008; 46:101-22. 